\title{
BMJ Open Efficacy and safety of left bundle branch area pacing versus biventricular pacing in heart failure patients with left bundle branch block: study protocol for a randomised controlled trial
}

\author{
Liting Cheng, ${ }^{1}$ Junmeng Zhang, ${ }^{1}$ Zefeng Wang, ${ }^{1}$ Mengge Zhou, ${ }^{2}$ Zhuo Liang, ${ }^{1}$ \\ Lin Zhao, ${ }^{1}$ Jieruo Chen, ${ }^{1}$ Yongquan Wu (i) ${ }^{1}$
}

To cite: Cheng L, Zhang J, Wang Z, et al. Efficacy and safety of left bundle branch area pacing versus biventricular pacing in heart failure patients with left bundle branch block: study protocol for a randomised controlled trial. BMJ Open 2020;10:e036972. doi:10.1136/ bmjopen-2020-036972

- Prepublication history and additional material for this paper are available online. To view these files, please visit the journal online (http://dx.doi org/10.1136/bmjopen-2020036972).

LC and JZ contributed equally.

Received 13 January 2020 Revised 11 July 2020 Accepted 25 August 2020
Check for updates

(c) Author(s) (or their employer(s)) 2020. Re-use permitted under CC BY-NC. No commercial re-use. See rights and permissions. Published by BMJ.

For numbered affiliations see end of article.

Correspondence to Dr Yongquan Wu; wuyongquan67@163.com

\section{ABSTRACT}

Introduction Left bundle branch area pacing (LBBaP) has been accepted as a physiological pacing method that can yield narrow paced QRS waves. For patients with failed biventricular pacing (Bi-V), LBBaP is another feasible option. However, no randomised controlled study has evaluated the efficacy and safety of LBBaP in heart failure patients with left bundle branch block (LBBB). Therefore, we aimed to conduct this type of randomised controlled trial.

Methods and analysis This study is a single-centre, randomised controlled non-inferiority trial. This study will be conducted at the cardiac centre of Beijing Anzhen Hospital. From January 2020 to December 2022, 180 heart failure patients with reduced left ventricular ejection fraction (LVEF $\leq 35 \%$ ) and LBBB undergoing Bi-V implantation will be consecutively enrolled in this study. Participants will be randomised at a 1:1 ratio into an experimental group (LBBaP) and a control group (Bi-V). The primary outcome is LVEF. The secondary outcomes are NT-proBNP, duration of the QRS complex, end systolic volume, end diastolic volume, the 6-minute walking test and quality of life (SF-36 scale), all causes of mortality, cardiovascular death, rehospitalisation rate of heart failure, other rehospitalisation rates, major complication rates, procedure costs and hospitalised dates. Ethics and dissemination This study has been approved by the Beijing Anzhen Hospital Medical Ethics Committee (No. ks201932). The results of this study will be presented at domestic and international conferences. We hypothesise that $\mathrm{LBBaP}$ is non-inferior compared with Bi-V for treating patients with heart failure and LBBB. This trial will provide evidencebased recommendations for electrophysiologists.

Trial registration number Chinese Clinical Trial Registry (ChiCTR2000028726).

\section{INTRODUCTION}

In 1932, Hyman ${ }^{1}$ first proposed the concept of a 'pacemaker'; since then, the discussion of the optimal position for implanting ventricular leads has been ongoing. His bundle pacing (HBP) is foreseen as the most physiological method. ${ }^{2}$ Compared with traditional right ventricular apex pacing, HBP significantly reduced the incidence
Strengths and limitations of this study

A randomisation sequence will be generated by a centralised computer system and a non-investigator is assigned to this job.

- Personnel will undergo training before this trial.

- A risk assessment team will evaluate the entire study.

- Three non-investigators will perform the data entry with EpiData.

- Experienced surgeons will perform the operations in turn.

of heart failure, mortality and morbidity. ${ }^{3}$ Furthermore, in patients with reduced systolic function and left bundle branch block (LBBB), HBP ameliorates quality of life.

During societal development, the incidence of heart failure has increased. ${ }^{4}$ Heart failure is the end stage of all kinds of cardiac diseases and is correlated with elevated rates of hospitalisation, mortality and morbidity. Heart failure is related to LBBB. ${ }^{5}$ As the cardiac conduction system works, LBBB results in left ventricular delays in contraction and left and right ventricle desynchronisation, which leads to heart failure. Biventricular pacing $(\mathrm{Bi}-\mathrm{V})$ is an advanced choice for correcting these conduction abnormalities. ${ }^{6}$

However, the procedure of implanting Bi-V is quite complex. For patients with venous malformations or coronary vein stenoses, left ventricular pacing leads are hard to implant. Moreover, Bi-V paces the right ventricle from the apex and the left ventricle from its free epicardium wall, which changes the systole and diastole in the heart. ${ }^{7}$ Last but not least, $\mathrm{Bi}-\mathrm{V}$ is expensive, demonstrating that a better implantation method is required. Therefore, a novel pacing method is needed to overcome those shortcomings. ${ }^{8}$ 
According to recent guidelines, both $\mathrm{HBP}$ and $\mathrm{Bi}-\mathrm{V}$ have become the indication for patients with an left ventricular ejection fraction (LVEF) between $36 \%$ and $50 \%$ who require ventricular pacing greater than $40 \%$ of the time or for patients with $\mathrm{AF}$ who undergo atrioventricular node ablation. ${ }^{9} \mathrm{HBP}$ is also recommended for patients after failure to implant biventricular in China. Due to the strengthening of HBP, electrophysiologists are exploring the indication for their use in cardiac resynchronisation therapy. His-SYNC Trial is the very first clinical study to compare HBP and Bi-V. ${ }^{10}$ Upadhyay et $a l^{11}$ conducted a secondary analysis on His-SYNC Trial and demonstrated that for heart failure patients, HBP has superior electrical resynchronisation than $\mathrm{Bi}-\mathrm{V}$. However, this randomised controlled trial failed for high rates of crossover.

Unfortunately, due to the specific anatomy characteristics of the His bundle, proximal HBP has a high pacing threshold and may cause abnormal sensing. Furthermore, HBP implantation can easily injure the bundle branch. In 2017, Huang et $a l^{12}$ developed the concept of LBBP. LBBP is a physiological pacing method that can yield narrower paced QRS waves and overcomes the shortcomings of $\mathrm{Bi}-\mathrm{V}$ and HBP. Moreover, LBBP may have other advantages over HBP, including lower thresholds, a higher $\mathrm{R}$ wave amplitude and is easier to implant.

To date, case reports have indicated that HBP and left bundle branch area pacing (LBBaP) are optimal strategies for patients who have failed Bi-V. ${ }^{13}$ For LBBaP, Zhang et al ${ }^{14}$ conducted an observational study where 11 patients with $\mathrm{Bi}-\mathrm{V}$ indications were treated with LBBaP, and the QRS duration was significantly shorter during pacing. However, a randomised controlled study is still lacking to evaluate the efficacy of LBBaP in heart failure patients with LBBB. Therefore, we aim to conduct this randomised controlled study. If successful, this study will provide evidence-based recommendations for electrophysiologists.

\section{RESEARCH QUESTIONS AND STUDY HYPOTHESIS \\ Objective}

The results of this trial will demonstrate whether LBBaP is non-inferior compared with Bi-V. This study will answer whether $\mathrm{LBBaP}$ is non-inferior than $\mathrm{Bi}-\mathrm{V}$ for the following characteristics: (1) LVEF, (2) NT-proBNP, (3) end systolic volume, (4) end diastolic volume, (5)6-minute walking test score, (6) quality of life (SF-36 scale), (7) duration of the QRS complex, (8) all causes of mortality, (9) cardiovascular death, (10) the rehospitalisation rate of heart failure, (11) other rehospitalisation rates, (12) major complication rates, (13) procedure costs and (14) hospitalised dates.

\section{Hypothesis}

We hypothesise that LVEF in LBBaP is non-inferior compared with $\mathrm{Bi}-\mathrm{V}$ in patients with heart failure and LBBB.

\section{METHODS AND ANALYSIS \\ Study design}

A single-centre, randomised controlled non-inferiority trial will be conducted at the cardiac centre of Beijing Anzhen Hospital. From January 2020 to December 2022, consecutive patients with heart failure and LBBB who undergo permanent pacemaker implantation will be enrolled in this study (figure 1).

This study has been approved by the Beijing Anzhen Hospital Medical Ethics Committee. Participants evaluated in this trial will sign an informed consent form (online supplemental file $1:$ Informed Consent) in which they will agree to participate in the trial while they are in the hospital as well as during follow-up.

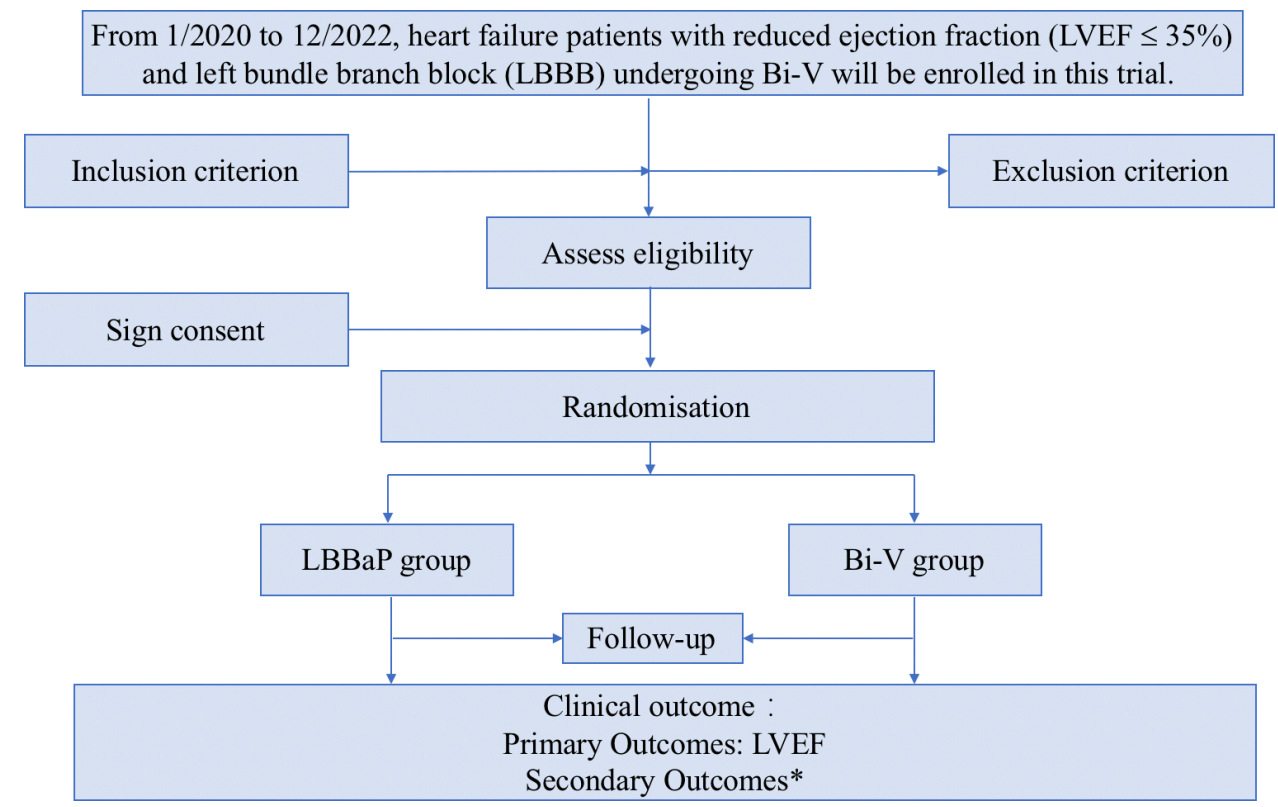

Figure 1 Flow diagram of enrolment, intervention and assessments. *Efficacy outcomes, safety outcomes and health economic outcomes. Bi-V, biventricular pacing; LBBaP, left bundle branch area pacing; LVEF, left ventricular ejection fraction. 


\section{Subjects}

Inclusion criteria

1. Adults aged $18-80$ years.

2. Meet American College of Cardiology Foundation (ACCF)/American Heart Association (AHA)/Heart Rhythm Society (HRS) class I or class II guideline indications for Bi-V. ${ }^{15}$

3. Left ventricular ejection fraction (LVEF) $\leq 35 \%$.

4. ECG indicates LBBB (under Strass criteria ${ }^{16}$ ).

5. Symptom cannot be improved by standard medical treatments.

6. Life expectancy of at least 1 year.

\section{Exclusion criteria}

1. Abnormal cardiac structure diseases (ie, severe mitral, tricuspid or aortic malformation; atrial or ventricular septal defects or tetralogy of Fallot).

2. History of radiofrequency catheter ablation (RFCA), cryoballoon ablation or cardiac surgical procedures.

3. Mental illness.

4. Estimated glomerular filtration rate $(e G F R)<30 \mathrm{~mL} /$ $\min$.

5. Septic shock.

6. Advanced malignant tumour.

7. Pregnancy or prepared to get pregnant.

8. Cardiac tamponade or major haemopericardium.

9. Patients without adequate veins.

\section{Study intervention}

\section{Bi-V group}

The left ventricular pacing lead will be implanted through the coronary vein. The right ventricular pacing lead will be implanted at the ventricular apex. After Bi-V pacing, the duration of the QRS complex will be narrow. If $\mathrm{Bi}-\mathrm{V}$ cannot be corrected, patients will undergo LBBaP implantation or surgery.

\section{LBBaP group}

\section{Implantation method}

The LBBaP lead will be implanted by 'New Nine Partition Method', details of the implantation technique have been discussed in an article written by our team. ${ }^{17}$ During the procedure, lead V1 signal is monitored by one bedside patient monitor. Lead $\mathrm{V}$ of the monitor is affixed to the V1 position for 12-lead ECGs. With the direction of the C315 sheath (Medtronic, Minneapolis, Minnesota, USA), the ventricular pacing lead (model 3830, Medtronic) will be inserted into the left bundle branch area. The threshold will be tested during the procedure by Medtronic pacemaker programmer. If LBBaP cannot be corrected, patients will undergo Bi-V implantation or surgery.

\section{Definition of a successful LBBaP}

1. 3830 lead was in the area of the LBB in the fluoroscopic image.

2. Unipolar pacing will demonstrate narrowed QRS complex $(<130 \mathrm{~ms}$ in the present study).
3. Stimulus to peak left ventricular activation time (S-pLVAT) (the duration from the stimulus to the peak of the $\mathrm{R}$ wave in leads $\mathrm{V} 4-\mathrm{V} 6$ ) will be $<90 \mathrm{~ms}{ }^{18}$

\section{Randomisation and masking}

A randomisation sequence will be generated by a centralised computer system; a non-investigator who will be blinded is assigned to this job. Sealed envelope will be used to conceal the sequence until interventions are assigned, subjects who fit the inclusion and exclusion criteria will be assigned to the $\mathrm{LBBaP}$ or Bi-V group at a 1:1 ratio.

\section{Study procedures \\ Screening}

Patients with heart failure and $\mathrm{LBBB}$ receiving treatment at the cardiac centre of Beijing Anzhen hospital from January 2020 to December 2022 will be screened with the inclusion and exclusion criteria. Once they meet the criteria, patients' baseline characteristics will be gathered using case report forms. Age, sex, body mass index (BMI), past history (ie, cardiomyopathy, coronary heart disease, hypertension and diabetes mellitus), medication history and blood sample test results will be included. Echocardiography and an electrogram will be evaluated by two experienced doctors separately. Interventricular thickness will be documented. The LVEF and QRS complex duration are essential factors to evaluate heart function and cardiac mechanical synchrony. A venous phase coronary angiogram in left anterior oblique and right anterior oblique will be used to assess the venous system and the presence of a posterolateral vein, for patients without adequate veins will not be enrolled.

\section{Data collection}

During the procedure, an intraoperative data collection form will be used. Information on complications, X-ray duration and the radiation dosage as well as detailed information on the pacemaker and pacing lead, pacemaker parameters (voltage, sensing, impedance, etc) and QRS duration will be observed.

\section{Randomisation and follow-up visits}

Randomisation will occur between 1 January 2020 and 31 July 2021, with an 18 month follow-up. Outpatient follow-up will be evaluated at 1, 3 and 18 months postoperation. Routine blood tests, echocardiography and electrograms will be performed. Particularly, ultrasound programmes to evaluate the A-V duration will be performed. Telephone follow-up will be performed at 6 and 12 months postoperation. The rehospitalisation rate will be mentioned (online supplemental table 1).

\section{Risk management}

A risk assessment team will evaluate the entire study. All mortality and morbidity events will be monitored. If patients develop any kind of cardiovascular disease, they will be admitted and a full-body test will be performed. If the initial outcome is unexpectedly bad or if there is a patient safety concern, we will stop this study. 


\section{OUTCOMES}

\section{Primary outcomes}

LVEF is critical for evaluating heart function. LVEF will be tested using echocardiography at 1, 3 and 18 months postoperation by experienced doctors.

\section{Secondary outcomes}

\section{Efficacy outcomes}

1. Short-term outcomes:

NT-proBNP, duration of the QRS complex, end systolic volume, end diastolic volume, the 6-minute walking test and quality of life (SF-36 scale).

2. Long-term outcomes:

All causes of mortality, cardiovascular death, rehospitalisation rate of heart failure and other rehospitalisation rates

\section{Safety outcomes}

Major complication rates: cardiac perforation, haemopericardium, malignant arrhythmias, sudden cardiac death and acute myocardial infarction

\section{Health economic outcomes}

Procedure costs (ie, pacemaker, leads, etc) and hospitalised dates

\section{Data management and monitoring}

Before the trial

Personnel will undergo training before this trial. Lectures will be given a week before this trial. Grouping will be performed by a special team. The operation team will uphold technical standards. The echocardiography measurement team will perform the measurements. The data processing team will use EpiData to perform the training and standardise the input using a Case Report Form (CRF) table. There will be no crossover among the randomly divided teams, including the operation and data entry teams. All personnel will sign a patient privacy confidentiality agreement.

\section{During the trial}

Patients will be randomly divided into two groups. Patients' crossover rate will be controlled in less than $10 \%$. Five surgeons will be randomised to perform the surgical treatment (only operators who had performed at least $50 \mathrm{Bi}-\mathrm{V}$ and $50 \mathrm{LBBaP}$ will perform the procedure). Three adjudicators will evaluate the relevant indicators, with two personnel collecting and collating the data and one person checking the data. There will be no crossover between the data collectors and the operators. Data will be anonymised with studied ID, stored and encrypted at Beijing Anzhen Hospital. It will be accessible to the personnel involved in this study.

\section{Interim analyses}

An interim analysis will be conducted at December 2020 to evaluate the complication rate and early signal for inferiority in LBBaP. If the complication rate is higher than $10 \%$, we will stop this trial.

\section{After the trial}

Research documents including the patient informed consent, CRF form, etc, will be collected and stored for a total of 10 years. Data set will be available by specific request through contacting the corresponding author (wuyongquan67@163. com).

\section{Sample size calculation}

This study is designed as a non-inferiority trial to evaluate the efficacy and safety of $\mathrm{LBBaP}$ in the treatment of patients with heart failure and LBBB. The main study outcome is the LVEF. According to previous results gathered by our team, the improvement of LVEF in the LBBaP group was 4.36-fold higher than in the traditional $\mathrm{Bi}-\mathrm{V}$ group at 3 months. The SD of LVEF in the LBBaP group was 10.84 and the SD of LVEF in the Bi-V group was 9.42. The non-inferiority margin was $1 / 5(0.87)$ of the difference between the two groups, which means that LBBaP is not inferior to Bi-V. Pass 11.0 (noninferiority test) was used to compare the sample size differences between the two groups. The significance level $(\alpha)$ was 0.025 , and the assurance level $(1-\beta)$ was 0.90 . At least 81 cases need to be enrolled in each of the two groups, with a total of 162 cases. Loss to follow-up is considered less than $10 \%$. To conclude, 180 cases will be enrolled. Beijing Anzhen Hospital is one of the largest cardiovascular centres in China. Consequently, this study has a large patient population.

\section{Statistical analysis}

Three non-investigators will perform the data entry with EpiData. Two independent statisticians will complete the statistical analysis. The Bi-V group was defined as follows: (1) complete Bi-V treatment or (2) failure to be treated with $\mathrm{Bi}-\mathrm{V}$ or eventually treated by LBBaP. Failure to be treated with $\mathrm{Bi}-\mathrm{V}$ is defined as follows: (a) diaphragm stimulus occurred at multiple points with the left ventricular electrode or (b) the left ventricular electrode pacing threshold was greater than $3 \mathrm{~V}$ at $0.4 \mathrm{~ms}$. The LBBaP group was defined as follows: (3) complete LBBaP treatment or (4) failure to be treated with $\mathrm{LBBaP}$ or eventually treated by $\mathrm{Bi}-\mathrm{V}$. Failure to be treated with $\mathrm{Bi}-\mathrm{V}$ is defined as follows: (a) LBBB could not be corrected; (b) Stiffness of the ventricular septum made it difficult to implant the ventricular lead or (c) the ventricular lead pacing threshold was greater than $3 \mathrm{~V}$ at $0.4 \mathrm{~ms}$. The intention-to-treat set is used to compare (1)+(2) with (3)+(4). The per-protocol analysis set is used to compare (1) with (3). The data will be analysed with SPSS statistics (IBM, V.23). Normally distributed continuous variables (age, sex, BMI, blood sample test results, NT-proBNP, duration of the QRS complex, LVEF, end systolic volume, end diastolic volume, the 6-minute walking test and SF-36 scale) will be expressed as the mean \pm SD and non-uniformly distributed data will be expressed as the median (Q1 and Q3). Comparison of means (past history, medication history) between groups will be analysed by the independent samples T-test for normally distributed data and the Mann-Whitney U-test for non-uniformly distributed data. A Kaplan Meier curve will be used to determine the events rate between the two groups over time, and the log rank test will be used to compare two groups. The 
Cox proportional risk model will be used to calculate the HR. A p value $<0.05$ will be considered statistically significant.

\section{Planned subgroup analyses}

1. Sex.

2. Ejection fraction.

3. Duration of QRS complex.

4. Six-minute walking test.

5. NYHA classification.

6. Quality of life (SF-36 scale).

\section{Bias}

Patients will be consecutively enrolled from the cardiac centre of Beijing Anzhen hospital. In total, 180 heart failure patients with reduced ejection fraction (LVEF $\leq 35 \%)$ and LBBB undergoing Bi-V will be included. Using a centralised computer system, participants will be assigned to the LBBaP or $\mathrm{Bi}-\mathrm{V}$ group at a 1:1 ratio.

\section{Patient and public involvement}

Patients or the public were not involved in the design, conduct, reporting or dissemination of the research.

\section{Ethics and safety considerations}

This study has been approved by the Beijing Anzhen Hospital Medical Ethics Committee (No 201932) and abides by the principles of the Helsinki Declaration. Participants evaluated in this trial will provide informed consent and sign an involvement statement in which they will agree to participate in the trial while they are in the hospital as well as during follow-up. All research documents will be collected and stored at Beijing Anzhen Hospital.

\section{Implementation and dissemination}

This single-centre, randomised controlled non-inferiority trial will be conducted at the cardiac centre of Beijing Anzhen Hospital from January 2020 to December 2022. After this trial, the results of this study will be shown at domestic and international conferences for research and further study.

\section{Trial status}

This protocol is version 3 . The trial will be started on 1 January 2020.

\section{Author affiliations}

${ }^{1}$ Department of Cardiology, Beijing Anzhen Hospital, Capital Medical University, Beijing, China

${ }^{2}$ Department of Epidemiology, Beijing Anzhen Hospital, Capital Medical University, the Key Laboratory of Remodeling-Related Cardiovascular Diseases, Ministry of Education, Beijing Institute of Heart, Lung and Blood Vessel Diseases, Beijing, China

Contributors LC, JZ, ZW, MZ, ZL, LZ, JC and YW involved in study design. LC and $\mathrm{JZ}$ contributed in drafting the manuscript. LC, JZ, ZW, MZ and YW critically revised the manuscript. All coauthors approved the final version for publication.

Funding Capital's Funds for Health Improvement and Research (CRF 2020-2-2062).

Competing interests None declared.

Patient and public involvement Patients and/or the public were involved in the design, or conduct, or reporting, or dissemination plans of this research. Refer to the 'Methods and analysis' section for further details.
Patient consent for publication Parental/guardian consent obtained.

Provenance and peer review Not commissioned; externally peer reviewed.

Open access This is an open access article distributed in accordance with the Creative Commons Attribution Non Commercial (CC BY-NC 4.0) license, which permits others to distribute, remix, adapt, build upon this work non-commercially, and license their derivative works on different terms, provided the original work is properly cited, appropriate credit is given, any changes made indicated, and the use is non-commercial. See: http://creativecommons.org/licenses/by-nc/4.0/.

ORCID iD

Yongquan Wu http://orcid.org/0000-0001-9156-4669

\section{REFERENCES}

1 Hyman AS. Resuscitation of the stopped heart by intracardial therapy II. experimental use of an artificial pacemaker. Arch Intern Med 1932;50:283-305.

2 Vijayaraman P, Dandamudi G, Zanon F, et al. Permanent his bundle pacing: recommendations from a multicenter his bundle pacing collaborative Working group for standardization of definitions, implant measurements, and follow-up. Heart Rhythm 2018;15:460-8.

3 Abdelrahman M, Subzposh FA, Beer D, et al. Clinical outcomes of His bundle pacing compared to right ventricular pacing. J Am Coll Cardiol 2018;71:2319-30.

4 Huerta-Preciado J, Franco J, Formiga F, et al. Differential characteristics of acute heart failure in very elderly patients: the prospective Rica study. Aging Clin Exp Res 2019;46:s40520-01901363-8.

5 Smiseth OA, Aalen JM. Mechanism of harm from left bundle branch block. Trends Cardiovasc Med 2019;29:335-42.

6 Vijayaraman P, Subzposh FA, Naperkowski A. Atrioventricular node ablation and his bundle pacing. Europace 2017;19:iv10-16.

7 Sharma PS, Vijayaraman P. His bundle pacing or biventricular pacing for cardiac resynchronization therapy in heart failure: discovering new methods for an old problem. J Atr Fibrillation 2016;9:1501.

8 Zhang S, Zhou X, Gold MR. Left bundle branch pacing: JACC review topic of the week. J Am Coll Cardiol 2019;74:3039-49.

9 Kusumoto FM, Schoenfeld MH, Barrett C, et al. 2018 ACC/AHA HRS Guideline on the Evaluation and Management of Patients With Bradycardia and Cardiac Conduction Delay: Executive Summary: A Report of the American College of Cardiology/American Heart Association Task Force on Clinical Practice Guidelines, and the Heart Rhythm Society. J Am Coll Cardiol 2019;74:932-87.

10 Upadhyay GA, Vijayaraman P, Nayak HM, et al. His Corrective Pacing or Biventricular Pacing for Cardiac Resynchronization in Heart Failure. J Am Coll Cardiol 2019;74:157-9.

11 Upadhyay GA, Vijayaraman P, Nayak HM, et al. On-Treatment comparison between corrective his bundle pacing and biventricular pacing for cardiac resynchronization: a secondary analysis of the His-SYNC pilot trial. Heart Rhythm 2019;16:1797-807.

12 Huang W, Su L, Wu S, et al. A novel pacing strategy with low and stable output: pacing the left bundle branch immediately beyond the conduction block. Can J Cardiol 2017;33:1736:1736.e1-1736.e3.

13 Dabrowski P, Kleinrok A, Kozluk E, et al. Physiologic resynchronization therapy: a case of His bundle pacing reversing physiologic conduction in a patient with CHF and LBBB during 2 years of observation. J Cardiovasc Electrophysiol 2011;22:813-7.

14 Zhang W, Huang J, Qi Y, et al. Cardiac resynchronization therapy by left bundle branch area pacing in patients with heart failure and left bundle branch block. Heart Rhythm 2019;16:1783-90.

15 Epstein AE, DiMarco JP, Ellenbogen KA, et al. 2012 ACCF/AHA/ HRS focused update incorporated into the ACCF/AHA/HRS 2008 guidelines for device-based therapy of cardiac rhythm abnormalities: a report of the American College of cardiology Foundation/American heart association Task force on practice guidelines and the heart rhythm Society. J Am Coll Cardiol 2013;61:e6-75.

16 Strauss DG, Selvester RH, Wagner GS. Defining left bundle branch block in the era of cardiac resynchronization therapy. Am J Cardiol 2011;107:927-34.

17 Zhang J, Wang Z, Zu L, et al. Simplifying physiological left bundle branch area pacing using a new nine partition method. Can J Cardiol 2020:j.cjca.2020.05.011.

18 Zhang J, Wang Z, Cheng L, et al. Immediate clinical outcomes of left bundle branch area pacing vs conventional right ventricular pacing. Clin Cardiol 2019;42:768-73. 